
oncology research (Springer), vol. 22 issue 3 (July 2016) p. 633-637. The final versión is available at DOI 10.1007/ s12253-015-9987-7

\title{
Protein kinase CK2 content in GL261 mouse glioblastoma
}

Laura Ferrer-Font ${ }^{1,2,3}$, Estefania Alcaraz ${ }^{1}$, Maria Plana ${ }^{1}$, Ana Paula Candiota ${ }^{2,1,3}$,

Emilio Itarte ${ }^{1}$, Carles Arús ${ }^{1,2,3}$

1. Departament de Bioquímica i Biologia Molecular, Unitat de Bioquímica de

Biociències, Edifici Cs, Universitat Autònoma de Barcelona, Cerdanyola del Vallès, Spain.

2. Centro de Investigación Biomédica en Red en Bioingeniería, Biomateriales y Nanomedicina (CIBER-BBN), Spain.

3. Institut de Biotecnologia i Biomedicina (IBB). Universitat Autònoma de Barcelona, Cerdanyola del Vallès, Spain.

ARTICLE TYPE: Communication

CORRESPONDING AUTHOR: Carles Arús (Carles.Arus@uab.cat)

Universitat Autònoma de Barcelona, 08193 Cerdanyola del Vallès, SPAIN

Phone +34 93581 1257, Fax +34935811264

Word count: 1750

Number figures and tables: 2

\section{ABSTRACT}

Glioblastoma (GBM) is the most prevalent and aggressive human glial tumour with a median survival of 14-15 months. Temozolomide (TMZ) is the standard chemotherapeutic choice for GBM treatment. Unfortunately, chemoresistence always ensues with concomitant tumour regrowth. Protein kinase CK2 (CK2) contributes to tumour development, proliferation, and suppression of apoptosis in cancer and it is overexpressed in human GBM. Targeting CK2 in GBM treatment may benefit patients. With this translational perspective in mind, we have studied the CK2 expression level 
by Western blot analysis in a preclinical model of GBM: GL261 cells growing orthotopically in C57BL/6 mice. The expression level of the CK2 catalytic subunit $(\mathrm{CK} 2 \alpha)$ was higher in tumour (about 4-fold) and in contralateral brain parenchyma (more than 2-fold) than in normal brain parenchyma $(\mathrm{p}<0.05)$. In contrast, no significant changes were found in CK2 regulatory subunit (CK2 $\beta$ ) expression, suggesting an increased unbalance of $\mathrm{CK} 2 \alpha / \mathrm{CK} 2 \beta$ in GL261 tumours with respect to normal brain parenchyma, in agreement with a differential role of these two subunits in tumours.

\section{KEY WORDS}

Glioma, CK2 content, preclinical brain tumor, GBM therapeutic targets

\section{ABBREVIATIONS LIST}

GBM: Glioblastoma

CK2: Protein kinase CK2

TMZ: Temozolomide

\section{INTRODUCTION}

Glioblastoma (GBM) is the most prevalent and aggressive glial tumour in humans with a median survival of 14-15 months even when an aggressive treatment is applied (surgical resection, chemotherapy and radiation) (1). Temozolomide (TMZ) plus radiotherapy is the standard therapeutic choice for GBM treatment and, at present, it is the one producing the best survival rates (2). Unfortunately, cancer stem cells (CSCs) are known to mediate chemoresistence, indicating that CSCs persist in these tumours even after the standard treatment (3). Due to its poor outcome and resistance to standard therapy, efficient alternative treatments are urgently needed for these tumours. 
The use of animal models in brain tumour research is mandatory in the search for new therapeutic targets due to obvious ethical restrictions related to human patients. The ideal animal model of GBM should reproduce the main characteristics of this neoplasm: characteristic growth pattern, infiltrative, but non-metastatic, tumour growth and poor immunogenicity (4). However, none of the currently available models is able to mimic all aspects of human high-grade glioma. One of the most investigated murine brain tumour models is GL261 growing into C57BL/6 mice, and it has been used for more than 20 years in different approaches in therapy evaluation studies (5). Furthermore, non-invasive biomarkers of therapy response have been evaluated in this model (6).

Protein Kinase $\mathrm{CK} 2$ is an oncogenic protein kinase which contributes to tumour development, proliferation, and suppression of apoptosis in multiple cancer types (7). It is composed of two catalytic ( $\alpha$ and/or $\alpha$ ) and two $\beta$ regulatory subunits. Protein Kinase CK2 is a constitutively active serine-threonine kinase and elevated CK2 expression levels have been demonstrated in several cancer types (8). It has been also demonstrated that it is overexpressed in mice xenograft models of human GBM (9) where it plays a fundamental role regulating multiple signalling pathways involved in tumour cell survival, proliferation, migration and invasion (10). These characteristics identify CK2 as an active therapeutic target, and in this sense, targeting CK2 in GBM treatment could benefit patients.

Although some authors have reported higher CK2 expression levels in tumours, in comparison with normal tissue $(8,11)$, to our knowledge this has not been studied in the 
GL261 GBM model. Accordingly, before evaluating CK2 inhibitors $(12,13)$ for therapy response studies we felt it advisable to assess the CK2 content in GL261 GBM.

\section{METHODS}

\section{$\underline{\text { Animal Models }}$}

A total of $6 \mathrm{C} 57 \mathrm{BL} / 6$ female wt mice weighting $18 \pm 2 \mathrm{~g}$ were used for this study. They were obtained from Charles River Laboratories (Charles River Laboratories Internacional, l'Abresle, France) and housed in the animal facility of the Universitat Autònoma de Barcelona. The GL261 mouse glioma cells were obtained from the Tumour Bank Repository of the National Cancer Institute, Frederick, MD, USA, and were cultured as described in (14). Tumours were induced in $3 \mathrm{C} 57 \mathrm{BL} / 6$ mice by intracranial stereotactic injection of $10^{5}$ GL261 cells in the caudate nucleus, as previously described by us (14).

Tumour volumes were followed using $\mathrm{T}_{2}$-weighted MRI acquisitions (see below and (6)), until the tumour reached the desired size, taking into account previous work from our group, that is $77.3 \pm 10.9 \mathrm{~mm}^{3}$ (15 days after implantation). Both tumour-bearing and control animals were euthanized with an intraperitoneal injection of pentobarbital (200 $\mathrm{mg} / \mathrm{kg}, 60 \mathrm{mg} / \mathrm{ml}$ ), their brains were removed and tumour resected (in case of tumourbearing animals). The brain parenchyma from three wt mice was used as control, while contralateral brain parenchyma was employed to determine the possible effects outside the tumour volume recently described by other authors (15). The contralateral tissue was obtained after visual inspection of the whole brain and tumour, avoiding as much as possible the tumoral zone. Samples were stored in liquid nitrogen until further processing. All studies were carried out following protocols approved by the 
local/institutional ethics committee, according to the regional and state legislation (CEEAH 2449 and 1176).

\section{$\underline{\text { In vivo MRI studies }}$}

These studies were carried out at the joint NMR facility of the Universitat Autònoma de Barcelona and CIBER-BBN (Cerdanyola del Vallès), using a 7T horizontal magnet (BioSpec 70/30, Bruker BioSpin, Ettlingen, Germany). $\mathrm{T}_{2}$-weighted images $(\mathrm{TR} / \mathrm{TEeff}=$ $4200 / 36 \mathrm{~ms}$ ) were acquired as in (6) to detect brain tumour evolution and to calculate the abnormal mass volume.

\section{$\underline{\text { Protein extraction }}$}

Tissue samples were weighted and $250 \mu \mathrm{l}$ of cold lysis buffer for each $100 \mathrm{mg}$ of tissue was added (cold lysis buffer: $50 \mathrm{mM}$ Tris-HClpH 7.7, $150 \mathrm{mM} \mathrm{NaCl}, 15 \mathrm{mM} \mathrm{MgCl}$, $0.4 \mathrm{mM}$ EDTA, $0.5 \mathrm{mM}$ DTT, $100 \mu \mathrm{g} / \mathrm{mL}$ Leupeptin, $100 \mu \mathrm{g} / \mathrm{mL}$ Aprotinin, $10 \mathrm{mM}$ Benzamidin, $2 \mathrm{mM}$ phenylmethylsulfonyl fluoride). Samples were homogenized with a Sonics sonicator (Vibra-cell, Newtown, USA) 10 times for 10-second intervals, and after, $0.5 \%$ Triton-X-100 was added. After remaining 15 min on ice, the lysate was centrifuged at $13,000 \times \mathrm{g}$ for $20 \mathrm{~min}$ at $4{ }^{\circ} \mathrm{C}$. Supernatants were used for Western blot analysis.

\section{$\underline{\text { Western blot analysis }}$}

The differential expression of protein $\mathrm{CK} 2 \alpha$ and $\mathrm{CK} 2 \beta$ in mice brain was assessed for control brain parenchyma, GL261 tumour and contralateral brain parenchyma (non tumoral). The protein content of tissue extracts was quantified with the Bradford assay (16). For Western blot analysis, $50 \mu \mathrm{g}$ of total protein were separated by $12 \%$ Sodium 
Dodecyl Sulfate Polyacrylamide Gel Electrophoresis (SDS-PAGE) and transferred onto Polyvinylidene fluoride (PVDF) membranes by electroblotting. The membranes were then blocked in 5\% milk powder in $0.02 \%$ Tween Phosphate buffered saline (T-PBS) for 1 hour and washed three times for 10 min with T-PBS. Membranes were incubated with antibodies against CK2 $\alpha$ (CK2 $\alpha$ (sc-6479 C-18), 42 kD, 1:300, Santacruz, Texas USA) or CK2 $\beta$ (CK2 $\beta$ EP1995Y, 24-25kD, 1:200, Merck Millipore, Darmstadt, Germany), and tubulin was used as a loading control ( $\beta$-tubulin, $55 \mathrm{kD}, 1: 1000$, Cell signalling technology (Danvers, Massachusetts, USA)) overnight at $4^{\circ} \mathrm{C}$. Next day, three T-PBS washing steps were carried out and incubation with the secondary antibodies took place during 1 hour (anti-goat for CK2 $\alpha$ and anti-rabbit for CK2 $\beta$ and tubulin, 1:5000, Biorad, Hercules, California) and washing steps with T-PBS were repeated. The specific antibody binding was detected with the enhanced chemiluminiscense technique with Lumi-Light Western blotting substrate (Roche, Basel, Germany). Western blot films were scanned and quantified with the ImageJ software (National Institutes of Health, USA, http://imagej.nih.gov/ij/) and data obtained was normalized to tubulin.

\section{$\underline{\text { Statistical analysis }}$}

Normality was first inspected in each group by the Kolmogorov-Smirnov test and variance homogeneity assessed with the Levene's test. A two-tailed Student's t-test for independent measurements was used for comparisons when data followed a normal Gaussian distribution. If data presented a non-normal distribution, Mann-Whitney U test was used for comparisons. The significance level for all tests was $\mathrm{p}<0.05$. 


\section{RESULTS AND DISCUSSION}

The expression level of CK2 catalytic subunit alpha (CK2 $\alpha)$ was about four-fold higher in tumour and more than two-fold higher in contralateral brain parenchyma than in normal brain parenchyma (figure 1 and table 1). On the other hand, no significant changes were detected in the expression of the CK2 regulatory subunit (CK2 $\beta)$, as shown in figure 1 , for selected samples, and quantitatively summarized in table 1. Furthermore, the here named "unbalancedness" ratio $\mathrm{CK} 2 \alpha / \mathrm{CK} 2 \beta$, significantly increased more than three-fold in tumours with respect to normal brain parenchyma.

\section{FIGURE 1 AND TABLE 1}

It has been consistently reported by other authors that high levels of CK2 $\alpha$ are expressed in solid human tumours (8), for example in colorectal carcinomas and breast carcinomas (11). Our results in preclinical GL261 GBM agree with (11), in the fact that we found higher expression levels of CK2 $\alpha$ in tumour, in comparison with the normal brain parenchyma. In our study, the Tumour/Normal brain parenchima ratio of CK2 $\alpha$ normalized expression values was of 3.88 (table 1). For CK2 $\beta$, a non significant increased expression was detected for the Tumour/ Normal brain parenchyma ratio (1.13).

A somewhat unexpected result found in our study was the significantly higher CK $2 \alpha$ expression level detected in the contralateral non-affected brain parenchyma, which has not been described in the literature as far as we are aware. In this respect, figures $1 \mathrm{~A}$ and 1B show a significantly higher $\mathrm{CK} 2 \alpha$ expression in contralateral tissue in comparison with the normal brain parenchyma, although this expression is clearly lower than the expression found in tumours (Table 1). This could be partially explained due to the well known infiltration pattern of the GBM, invading adjacent non-tumoral tissue 
and even producing a precancerous stage in apparently uninvolved parenchyma (15). In this respect, Lama and collaborators (17) also described an increased ERK1/2 expression in GBM peritumoral tissue from patients. Taking into account that ERK1/2 seems to be activated for translocation to the nucleus by CK2 phosphorylation (18) our results on the increased CK2 content in the contralateral/peritumoral area would agree with those reports and reinforce the idea of a "precancerous stage" away from the tumour mass, also for the CK2 content.

Regarding the different $\mathrm{CK} 2$ subunits evaluated (CK2 $\alpha$ and $\mathrm{CK} 2 \beta)$, our results suggest that an unbalanced expression is taking place in the GL261 GBM (see Figures 1A, 1B and table 1), with a predominance of an overexpression of CK2 $\alpha$ in the studied tumours. This imbalance of CK2 subunits was also seen in (18), being produced in this case by a relative decrease in the CK2 $\beta$ regulatory subunit in biopsies of human breast cancer tumours having suffered epithelial-to-mesenchymal transition, a sign of increased aggressivity.

Accordingly, the high expression levels observed for CK2 $\alpha$ in GBM, in comparison with normal brain parenchyme, reinforces the potential of $\mathrm{CK} 2 \alpha$ as a valid therapeutic target $(19,20)$ to investigate in GL261 GBM. In this sense, recent studies have reported that promiscuous CK2 inhibitors such as apigenin have shown relevant effects in the growth inhibition of established human GBM cultured cells lines (9). Additionally, a more specific CK2 inhibitor, CX-4945, has been reported as the first CK2 inhibitor to reach clinical stage testing for the treatment of multiple types of cancer $(13,20)$. Moreover, there are other promising CK2 inhibitors in development, such as tetra- 
bromo-deoxyribofuranosyl-benzimidazole (TDB) which is a dual inhibitor of CK2 and Proviral Integration of Moloney virus (PIM-1) (12).

Finally, the preclinical GL261 mice GBM model has been widely used for molecular imaging studies and to evaluate TMZ therapy response. Accordingly, the results here described warrant further work with $\mathrm{CK} 2 \alpha$ inhibition in this GBM model as standalone or combination therapeutic strategies.

\section{CONCLUSIONS}

The expression level of the CK2 catalytic subunit (CK2 $\alpha$ ) was found higher in GL261 GBM tumour (about 4-fold) and in contralateral brain parenchyma (more than 2-fold) than in normal brain parenchyma $(\mathrm{p}<0.05)$. In contrast, no-significant changes were found in the CK2 regulatory subunit (CK2 $\beta$ ) expression, suggesting a predominance of CK2 $\alpha$ overexpression in the evaluated tumours and possibly pointing to a differential role for the two subunit types in GBM tumour development in our investigated model. This should also be taken into account in future studies with preclinical GBM and therapeutic CK2 targeting.

\section{ACKNOWLEDGMENTS}

This work was supported by: SAF 2011-23870, SGR191-2014 and Centro de Investigación Biomédica en Red - Bioingeniería, Biomateriales y Nanomedicina (CIBER-BBN, [http://www.ciber-bbn.es/en]), an initiative of the Instituto de Salud Carlos III (Spain) co-funded by EU FEDER funds. 


\section{CONFLICT OF INTEREST DICLOSURES}

The authors have no financial conflicts to disclose. 


\section{REFERENCES}

1. Buckner JC. Factors influencing survival in high-grade gliomas. Semin Oncol. 2003;30(6 Suppl (19):10-4.

2. Stupp R, Mason WP, van den Bent MJ, Weller M, Fisher B, Taphoorn MJ, et al. Radiotherapy plus concomitant and adjuvant temozolomide for glioblastoma. N Engl J Med. 2005 Mar 10;352(10):987-96.

3. Huang Z, Cheng L, Guryanova OA, Wu Q, Bao S. Cancer stem cells in glioblastoma - molecular signalling and therapeutic targeting. Protein Cell. 2010;1(7):638-55.

4. Ausman JI, Shapiro WR, Rall DP. Studies on the chemotherapy of experimental brain tumors: Development of an Experimental Model. Cancer Res. 1970;30:2394-400.

5. Szatmári T, Lumniczky K, Désaknai S, Trajcevski S, Hidvégi EJ, Hamada H, et al. Detailed characterization of the mouse glioma 261 tumor model for experimental glioblastoma therapy. Cancer Sci. 2006;97(6):546-53.

6. Delgado-Gỗi T, Julià-Sapé M, Candiota AP, Pumarola M, Arús C. Molecular imaging coupled to pattern recognition distinguishes response to temozolomide in preclinical glioblastoma. NMR Biomed 2014;27(11):1333-45.

7. Ducan JS, Litchfield DW. Too much of a good thing: The role of protein kinase CK2 in tumorigenesis and prospects for therapeutic inhibition of CK2. Biochim Biophys Acta. 2008;1784:33-47.

8. Ruzzene M, Pinna LA. Addiction to protein kinase CK2: A common denominator of diverse cancer cells? Biochim Biophys Acta. 2010;1804(3):499-504.

9. Dixit D, Sharma V, Ghosh S, Mehta VS, E. S. Inhibition of Casein kinase-2 induces p53-dependent cell cycle arrest and sensitizes glioblastoma cells to tumor 
necrosis factor (TNF $\alpha)$-induced apoptosis through SIRT1 inhibition. Cell Death Dis. 2012;3:e271.

10. Ji H, Lu Z. The role of protein kinase CK2 in glioblastoma development. Clin Cancer Res. 2013;19:6335-7.

11. Münstermann U, Fritz G, Seitz G, Yiping L, Schneider HR, Issinger OG. Casein Kinase II is elevated in solid human tumours and rapidly proliferating non-neoplastic tissue. Eur J Biochem. 1990;189:251-7.

12. Cozza G, Girardi C, Ranchio A, Liolli G, Sarno S, Orzeszko A, et al. Cellpermeable dual inhibitors of protein kinases CK2 and PIM-1: structural features and pharmacological potential. Cell Mol Life Sci. 2014;71:3173-85.

13. Kim J, Hwan Kim S. CK2 inhibitor CX-4945 blocks TGF-beta1- induced epithelial-to mesenchymal transition in A549 human lung adenocarcinoma cells. PLoS ONE. 2013;8(9):e74342.

14. Simoes RV, Garcia-Martin ML, Cerdan S, Arus C. Perturbation of mouse glioma MRS pattern by induced acute hyperglycemia. NMR Biomed. 2008 Mar;21(3):251-64.

15. Mangiola A, Saulnier N, De Bonis P, Orteschi D, Sica G, Lama G, et al. Gene expression profile of glioblastoma peritumoral tissue: An ex vivo study. PLoS ONE. 2013;8:e57145.

16. Bradford MM. A rapid and sensitive method for the quantitation of microgram quantities of protein utilizing the principle of protein-dye binding. Anal Biochem. $1976 ; 72: 248-54$.

17. Lama G, Mangiola A, Anile C, Sabatino G, De Bonis P, Lauriola L, et al. Activated ERK1/2 expression in glioblastoma multiforme and peritumor tissue. Int $\mathrm{J}$ Oncol. 2007;30:1333-42. 
18. Plotnikov A, Chuderland D, Karamansha Y, Livnah O, Seger R. Nuclear Extracellular Signal-Regulated Kinase 1 and 2 translocation is mediated by Casein Kinase 2 and accelerated by autophosphorylation. Mol and Cel Biology. 2011;31(17):3515-30.

19. Agarwal M, Nitta RT, Li G. Casein kinase 2: A novel player in glioblastoma therapy and cancer stem cells. Mol Genet Med. 2014;8:pii: 1000094.

20. Zheng Y, McFarland BC, Drygin D, Yu H, Bellis SL, Kim H, et al. Targeting protein kinase CK2 supresses prosurvival signaling pathways and growth of glioblastoma. Clin Cancer Res. 2013;19(23).

\section{FIGURE LEGENDS}

Figure 1. Immunoquantification of CK2 subunits in mouse brain tumour samples.

(A) Western blots of characteristic tissue samples using $50 \mu \mathrm{g}$ of total protein, detection with anti-CK2 $\alpha$ antibody, anti-CK2 $\beta$ antibody and with anti-tubulin antibody. From left to right, normal brain parenchyma samples, tumour samples and contralateral brain parenchyma samples. (B) Quantification (bar; ${ }_{+} \mathrm{SD}$ ) carried out of the normalized CK2 $\alpha$ to tubulin expression in tissue samples using $50 \mu g$ of total protein, $n=9$. N=normal brain parenchyma, $T=$ tumour, $C L=$ contralateral brain parenchyma $*=p<0.05$.

Table 1. Expression values for CK2 $\alpha$ and CK2 $\beta$ and their ratios in mouse brain tumour samples.

Normalized expression values $( \pm S D)$ and their $T / N, C L / N$ and $T / C L$ ratios $( \pm S D)$ of the quantification carried out for $C K 2 \alpha$ and $C K 2 \beta, n=9$. Abbreviations as in figure 1 
legend. (*) significance compared with $N,(¥)$ significance compared with $T,(£)$ significance compared with T/N and (\$) significance compared with CL/N.

\section{TABLES}

Table 1:

\begin{tabular}{|c|c|c|c|c|c|c|c|c|c|}
\hline \multicolumn{4}{|c|}{ CK2a } & \multicolumn{4}{|c|}{ CK2 $\beta$} & \multicolumn{2}{|c|}{ CK2 $\alpha / C K 2 \beta$} \\
\hline $\mathbf{N}$ & $0.32 \pm 0.1^{\mp}$ & $\mathbf{T} / \mathbf{N}$ & $3.88 \pm 1.3^{t}$ & $\mathbf{N}$ & $1.07 \pm 0.4$ & $\mathbf{T} / \mathbf{N}$ & $1.13 \pm 0.6$ & $\mathbf{N}$ & $0.30 \pm 0.15^{7}$ \\
\hline $\mathbf{T}$ & $1.24 \pm 0.1^{*}$ & CL/N & $2.51 \pm 0.8^{8}$ & $\mathbf{T}$ & $1.21 \pm 0.4$ & CL/N & $1.03 \pm 0.5$ & $\mathbf{T}$ & $1.02 \pm 0.4^{*}$ \\
\hline CL & $0.8 \pm 0.1^{* ¥}$ & T/CL & $1.55 \pm 0.2^{ \pm \$}$ & $\overline{C L}$ & $1.1 \pm 0.3$ & T/CL & $1.09 \pm 0.5^{t}$ & $\mathbf{C L}$ & $0.73 \pm 0.2^{* \#}$ \\
\hline
\end{tabular}

\section{FIGURES:}

A
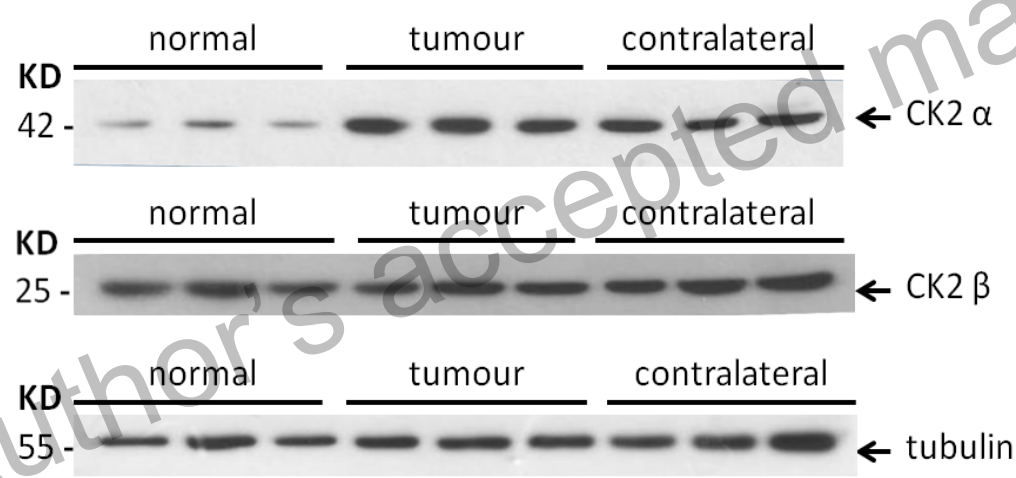

B

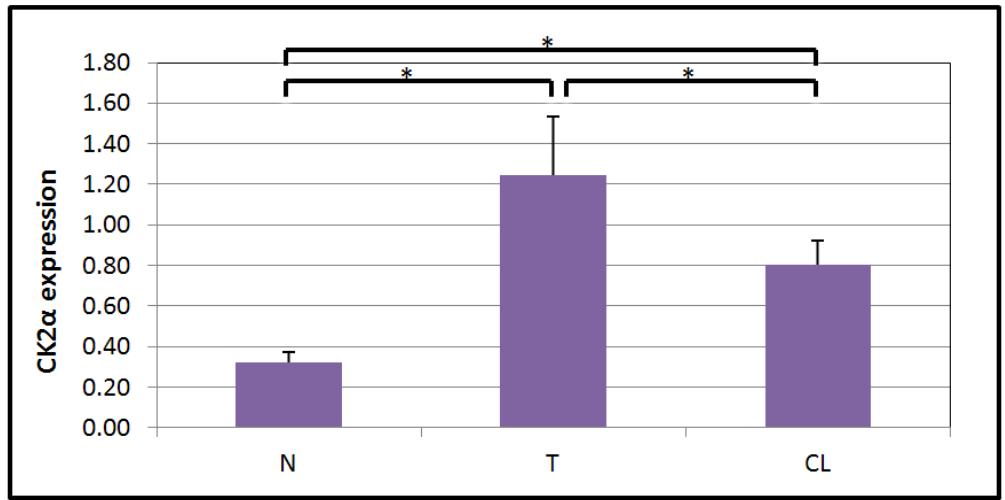




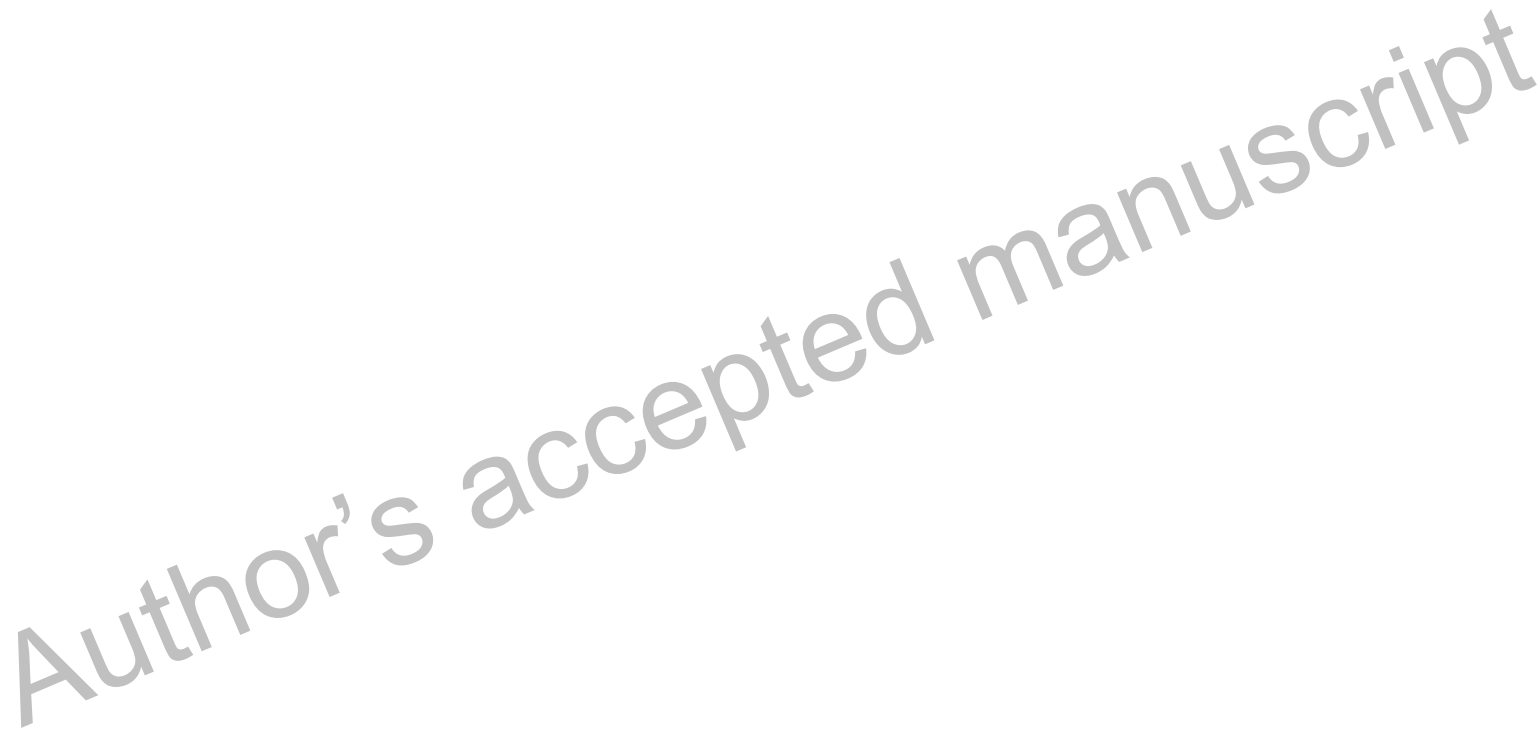


A

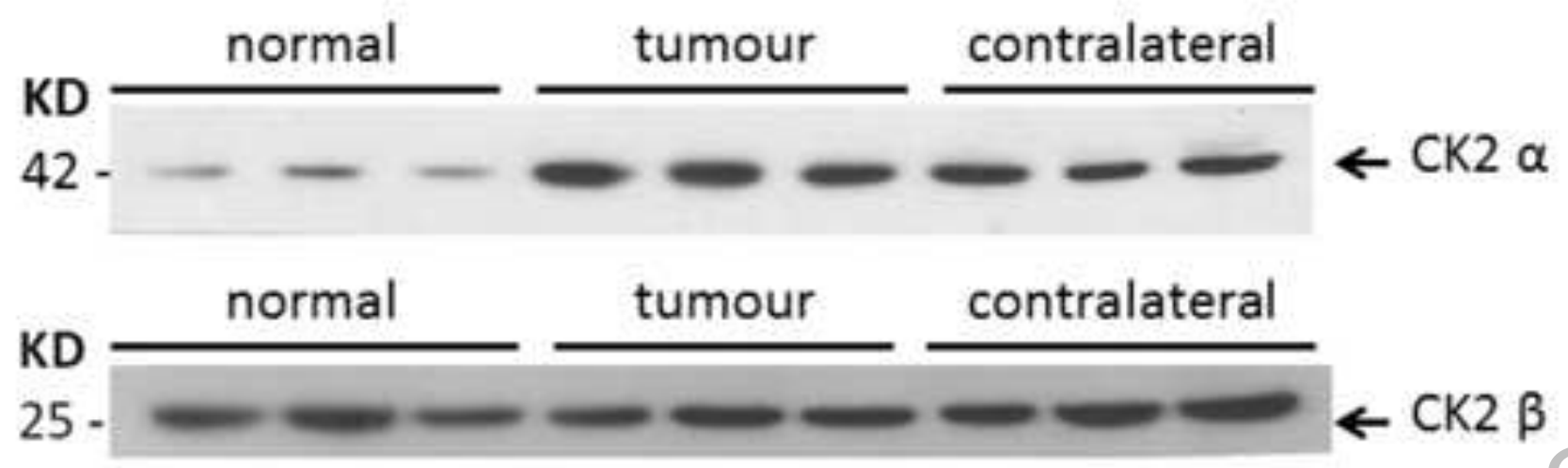

KD

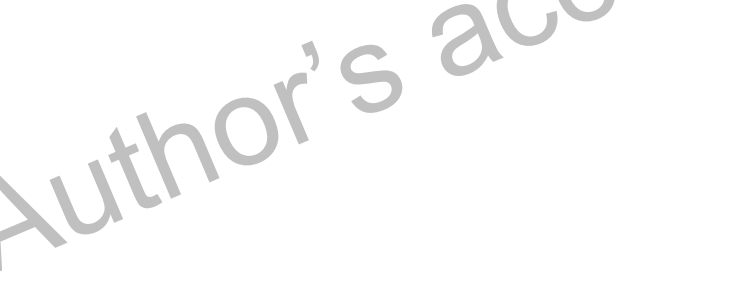


Click here to download high resolution image

B

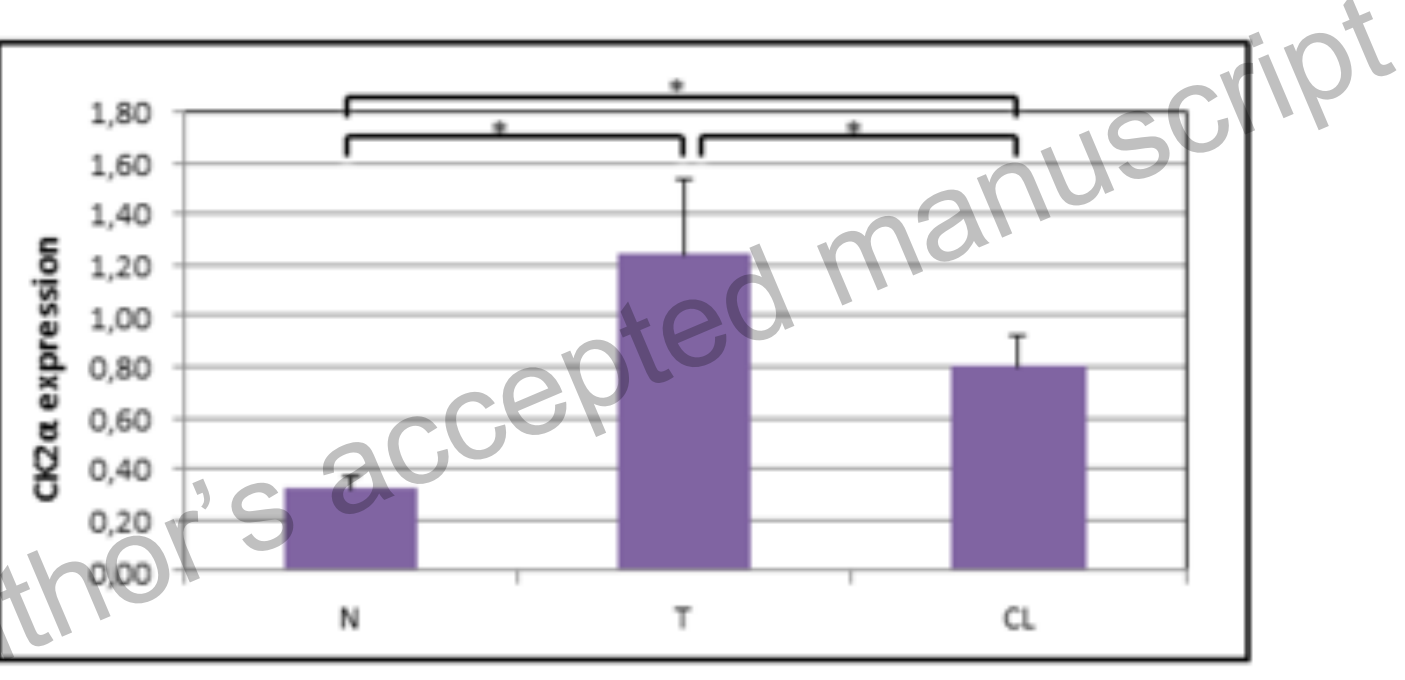

